\title{
Contaminação, por ovos de Tox ocara spp, de parques e praças públicas de Botucatu, São Paulo, Brasil
}

\author{
Contamination by Toxocara spp eggs in public parks and \\ squares in Botucatu, São Paulo State, Brazil \\ Vamilton Alvares Santarém, Izidoro Francisco Sartor \\ e Fabiana Mitie Matsubara Bergamo
}

Resumo A freqüência de contaminação de parques e praças públicas de Botucatu, São Paulo, Brasil, por ovos de Toxocara spp foi estudada durante 12 meses, com colheitas mensais de amostras de solo de dez praças, que foram processadas pela técnica de concentração em solução decinormal de hidróxido de sódio. Das 120 amostras analisadas, 21 estavam contaminadas, correspondendo a 17,5\%, em um total de seis praças. A maioria desses ovos porém apresentaram características de inviabilidade infectiva. Embora a chance de aquisição dessa zoonose nas praças estudadas seja pequena, existe o risco de infecção da população. Palavras-chaves: Toxocara spp. Parques e praças públicas. Contaminação. Epidemiologia.

\begin{abstract}
The frequency of Toxocara spp eggs in public parks was determined from March 1995 to February 1996. One hundred and twelve samples were collected from 10 public parks in Botucatu, São Paulo State, Brazil. Samples were processed by the decinormal sodium hydroxide concentration method. Out of the 120 soil samples analyzed, 21 were contaminated with Toxocara spp eggs, corresponding to a $17.5 \%$ rate of infestation. Of the ten squares submitted to analysis during the year, six presented contamination. Most of the ova found presented characteristics of inviability, with a consequent low chance of human infection, although the population is not free from the risk of contracting this zoonosis.
\end{abstract}

Key-words: Toxocara spp. Public parks. Contamination. Epidemiology.

\footnotetext{
Laboratório de Enfermidades Parasitárias da Faculdade de Medicina Veterinária e Zootecnia da Universidade Estadual Paulista, Campus de Botucatu, Botucatu, SP.

Financiado pela Fundo de Amparo à Pesquisa do Estado de São Paulo - FAPESP

Endereço para correspondência: Dr. Izidoro Francisco Sartor. Dpto. de Clínica Veterinária/FMVZ/UNESP. Caixa Postal 560, Distrito de Rubião Jr, 18618-000 Botucatu, SP, Brasil.

Fax: (014) 821-3743.

Recebido para publicação em 10/12/97.
} 
Toxocara spp, particularmente o Toxocara canis, em seus estágios larvais, por migração errática, ocasionam importante zoonose. É considerado como um dos mais comuns parasitos mundiais, cuja prevalência pode chegar até $81 \%$ da população de cães 23 . Em estudos sorológicos em humanos, a freqüência de anticorpos anti-Toxocara atingiu 7\% da população nos Estados Unidos, 5\% no Canadá, e 3,6\% em São Paulo?.

A infecção humana é mais freqüente em crianças com idade entre um e cinco anos, cujos hábitos geofágicos levam-nas a ingerirem ovos embrionados de Toxocara spp presentes em ambientes contaminados. Estas larvas eclodirão no intestino e migrarão pela via linfática ou circulação portal para diversos órgãos, principalmente fígado e pulmões; e, ocasionalmente, coração e sistema nervoso central, originando a síndrome Larva migrans visceral 4 , ou afetando o globo ocular e gerando a síndrome Larva migrans ocular 27.

Epidemiologicamente é relevante considerar que cães e gatos, pelo livre acesso a locais de recreação, contaminem o solo16, eliminando até 15.000 ovos por grama de fezes 2 . Esses ovos, devido a consistência de sua cutícula externa, permanecem viáveis por longo período no ambiente6, expondo a população humana ao risco de infecção e desenvolvimento da doença.

Muitos autores têm realizado estudos sobre a contaminação de solos por ovos de Toxocara spp (Tabela 1).

Tabela 1 - Prevalência de contaminação de praças por ovos de Toxocara spp em alguns países.

\begin{tabular}{|c|c|c|}
\hline País & Número de amostras examinadas & Freqüência (\%) \\
\hline Jordania1 & 194 & 0,2 \\
\hline Brasil3 & 298 & 24,8 \\
\hline Reino Unido5 & - & 24,4 \\
\hline Brasil8 & 15 & 60,0 \\
\hline Nigéria9 & 100 & 13,0 \\
\hline Brasil10 & 24 praças & 91,7 \\
\hline Estados Unidos 11 & 50 & 16,0 \\
\hline Estados Unidos 12 & - & 12,5 \\
\hline Alemanha ${ }^{13}$ & 31 praças & 87,0 \\
\hline Canadá14 & 43 & 32,5 \\
\hline Índia15 & - & 6,6 \\
\hline Estados Unidos 16 & 114 & 19,0 \\
\hline Irlanda18 & 228 & 15,0 \\
\hline Inglaterra19 & 14 & 13,3 \\
\hline Portuga|20 & 23 praças & 40,0 \\
\hline Japão21 & 46 & 87,5 \\
\hline Inglaterra22 & 503 & 66,0 \\
\hline Estados Unidos24 & 629 & 0,3 \\
\hline Japão25 & 13 praças & 92,0 \\
\hline Iraque26 & - & 25,0 \\
\hline
\end{tabular}

\section{MATERIAL E MÉTODOS}

Escolha das amostras e período de colheita. Dez parques e praças públicas do município de Botucatu, São Paulo, contendo caixas de areia ou brinquedos infantis, foram utilizados para a colheita de amostras de solo, mensalmente, por um período de 12 meses.

Colheita de amostras. As amostras foram colhidas aleatoriamente, com a ajuda de uma pá-de-pedreiro, em cinco diferentes áreas de cada praça com uma distância mínima de cinco metros de distância de qualquer material fecal.
A camada superfícial do solo era retirada para remoção de sujidades e o material, aproximadamente 250 gramas, foi obtido de uma profundidade de até oito centímetros, sendo então colocado em um saco plástico e mantido sob refrigeração até a análise laboratorial.

Técnica de recuperação de ovos. Amostras de solo foram tamisadas em peneiras de diversas malhas até a obtenção de um fino pó. Do material resultante, cinco gramas foram colocados em um tubo cônico de centrífuga, processados pela 
técnica de flutuação em solução decinormal de hidróxido de sódio ${ }^{11}$, e observados à microscopia ótica (10x) com três repetições do processamento para cada amostra.

\section{RESULTADOS}

O percentual de amostras para ovos de Toxocara spp está representado na Tabela 2.

Das 120 amostras analisadas, em 21 delas observou-se a presença de ovos de Toxocara spp, correspondendo a $17,5 \%$. Das praças e parques submetidos ao estudo, seis apresentaram contaminação, totalizando $60 \%$. Outros parasitos encontrados no solo foram as larvas de ancilostomídeos e cistos de Isospora spp, ambos em $0,8 \%$ das amostras de uma única praça, que apresentou também a maior freqüência de ovos $(70 \%)$.

Tabela 2- Freqüência de ovos de Toxocara spp em solo de dez praças e parques públicos de Botucatu, São Paulo, Brasil.

\begin{tabular}{|c|c|c|c|c|}
\hline \multicolumn{2}{|c|}{ Positivo } & \multicolumn{2}{|c|}{ Negativo } & \multirow{2}{*}{$\begin{array}{c}\text { Total } \\
\mathrm{n}^{-} \\
\end{array}$} \\
\hline $\mathrm{n}^{\circ}$ & $\%$ & $\mathrm{n}^{0}$ & $\%$ & \\
\hline 21 & 17,5 & 99 & 82,5 & 120 \\
\hline
\end{tabular}

\section{DISCUSSÃO}

É evidente o risco de infecção humana por ovos de Toxocara spp, particularmente em crianças que freqüentam praças e parques públicos, onde é comum a presença de animais.

Em Botucatu, os níveis de contaminação de parques e praças públicas com ovos de Toxocara spp são semelhantes aos resultados verificados por outros autores3 5911161926 , que registraram graus de contaminação em 13 a $25 \%$ das amostras.

Das amostras analisadas individualmente o número de ovos nunca foi superior a três e, daqueles recuperados, apenas dois apresentaram características morfológicas consideradas como normais. Esses achados divergem daqueles encontrados por alguns autores 1132125 , que observaram pelo menos $35,4 \%$ de ovos infectivos, ressaltando-se porém que nesses ensaios o material processado continha fezes misturadas ao solo.
Verificou-se uma maior recuperação de ovos nos meses de primavera e verão, coincidindo com os dados apresentados por Shimizu21. A grande redução de contaminação nos meses secos poderia ser explicado pela influência das baixas umidades sobre a manutenção de ovos no solo, corroborando com as afirmações de Borg \& Woodruff5.

Apesar do grande número de praças contaminadas, a chance de aquisição dessa zoonose nesses locais torna-se reduzida pelo alto número de ovos com características de inviabilidade. Esse fato deveu-se, provavelmente, às condições climáticas do município assim como aos tipos de solo existentes nos locais de colheita de amostras, que devem facilitar a dissecação dos ovos, fenômeno este também verificado por Nunes et al17.

\section{REFERÊNCIAS BIBLIOGRÁFICAS}

1. Abo-shehada MN. Prevalence of Toxocara ova in some schools and public grounds in northern and central Jordan. Annals of Tropical Medicine and Parasitology 83:73-75, 1989.

2. Acha PN, Szyfres B. Zoonosis y enfermedades transmisibles al hombre y a los animales, 2nd edition, Organización Mundial de la Salud, Washington, 1986.

3. Alcântara N, Bavia E, Silvão RM, Carvalho E. Environmental Contamination by Toxocara sp eggs in public areas of Salvador, Bahia State, Brazil. Revista da Sociedade Brasileira de Medicina Tropical 22:187190, 1989.

4. Beaver PC, Snyder CH, Carrera GM, Dent JH, Lafferty JW. Chronic eosinophilia due to visceral larva migrans. Pediatrics 9:7-19, 1952.
5. Borg OA, Woodruff, AW. Prevalence of infective ova of Toxocara species in public places. British Medical Journal 4:470-472, 1973.

6. Bourdeau P. Toxocara canis: infestation du chien et de I'homme, méthodes de lutte. Point Veterinary 18:551-564, 1986.

7. Chieffi PP. Contribuição ao estudo da síndrome de Larva migrans causada por larvas de Toxocara (Stiles e Hassal, 1905), em cinco Municípios do Estado de São Paulo, Brasil: inquérito soroepidemiológico. Tese de Doutorado, Universidade de São Paulo, São Paulo, SP, 1984.

8. Chieffi PP, Muller EE. Prevalência de parasitismo por Toxocara canis em cães e presença de ovos de Toxocara sp no solo de localidades públicas da zona 
urbana do município de Londrina, Estado do Paraná Brasil. Revista de Saúde Pública 20:367-372, 1976.

9. Chiejina SN, Ekwe TO. Canine Toxocariasis and associated Environmental Contamination of Urban Areas in Eastern Nigeria. Veterinary Parasitology 22:157-161, 1986.

10. Corrêa GLB, Michelon E, Lagaggio VRA, Moreira WS, Moraes RQ, Leite CR, Ribas HO, Adamy M, Pit $\mathrm{GL}$, Colombo FH. Contaminação do solo por ovos, larvas de helmintos e oocistos de protozoários, em praças públicas de Santa Maria e sua importância em saúde pública. Revista Brasileira de Parasitologia Veterinária 4:137, 1995.

11. Dada BJO, Lindquist WD. Prevalence of Toxocara spp eggs in some publc grounds and highway rest areas in Kansas. Journal of Helmintology 53:145146, 1979.

12. Dubin S, Segall S, Martindale J. Contamination of soil in two city parks with canine nematode ova including Toxocara canis: a Preliminary Study. American Journal of Public Health 65:1242-1245, 1975.

13. Düwell D. The prevalence of Toxocara eggs in the sand in children's playgrounds in Frankfurt/M. Annals of Tropical Medicine and Parasitology 78:633-636, 1984.

14. Ghadirian E, Viens P, Strykowski M, Dubreuil UIL F. Epidemiology of Toxocariasis in the Montreal area, prevalence of Toxocara and other helminth ova in dogs and soil. Canadian Journal of Public Health 67:495-498, 1976.

15. Gunasselan L, Ganesan PI, Ramadass P, Basheer A, Raghavan N. Incidence of Toxocara ova in the environment. Indian Veterinary Journal 69:308-309, 1992.

16. Ludlam KE, Platt TR. The relationship of park maintenance and accessibility to dogs to the presence of Toxocara spp ova in the soil. American Journal of Public Health 79:633-634, 1989.

17. Nunes CM, Sinhorini IL, Ogassawara S. Influence of soil texture in the recovery of Toxocara canis eggs by a flotoation method. Veterinary Parasitology 53:269274, 1994.
18. O'Lorcain P. Prevalence of Toxocara canis ova in public playgrounds in the Dublin area of Ireland. Journal of Helmintology 68:237-241, 1994.

19. Read MA, Thompson CA. Prevalence of Toxocara canis and Toxascaris leonina ova in dog faeces deposited on the streets of Leeds. Journal of Helmintology 50:95-96, 1976.

20. Rego AA. Contaminação do solo de parques e praças de Lisboa por ovos de Toxocara e outros helmintos. Anais da Escola Superior de Medicina Veterinária Lisboa 22:153-162, 1980.

21. Shimizu T. Prevalence of Toxocara eggs in sandpits in Tokushima City and its Outskirts. Journal of Veterinary Medicine Science 55:807-811, 1993.

22. Snow KR, Ball SJ, Bewick JA. Prevalence of Toxocara spp eggs in the soil of five east London parks. Veterinary Record 120:66-67, 1987.

23. Soulsby EJL. Ascaris In: Soulsby EJL (ed) Helmints, Arthropods and Protozoa of Domesticated Animals. $7^{\text {th }}$ edition, Lea \& Febiger, Philadelphia, p. 143-154, 1982.

24. Surgan MH, Colgan KB, Kennet SI, Paffmann JV. A survey of canine toxocariasis and toxocaral soil contamination in Essex County, New Jersey. American Journal of Public Health 70:1207-1208, 1980.

25. Uga S. Prevalence of Toxocara eggs and number of faecal deposits from dogs and cats in sandpits of public parks in Japan. Journal of Helmintology 67:7882, 1993.

26. Woodruff AW, Watson J, Shikara J, Al Azzi SH, Al Hadithi TS, Al Adhami SBH, Woodruff PNR. Toxocara ova in soil in the Mosul District, Iraq, and their relevance to public health measures in the Middle East. Annals of Tropical Medicine and Parasitology 75:555-557, 1981.

27. Zinkham $\mathrm{WH}$. A review and reassessment indicating two forms of clinical expression: visceral and ocular. American Journal Diseases of Children 132:627-628, 1978. 